\title{
Many perinatal factors increased the risk of developmental dysplasia of the hip
}

\author{
Chan A, McCaul KA, Cundy PJ, et al. Perinatal risk factors for developmental dysplasia of the hip. Arch Dis Child Fetal Neonatal Ed \\ 1997 Jan;76:F94-100.
}

\section{Objective}

To determine perinatal risk factors for developmental dysplasia of the hip (DDH).

\section{Design \\ Case control study.}

\section{Setting}

A community based study in South Australia.

\section{Participants}

1127 children who were born from 1986-93 and had DDH were compared with 150130 children who did not have DDH and were born during the same time period. Children with major abnormalities were excluded.

\section{Assessment of risk factors}

Data from state birth records were used to ascertain mother's age, race, country of birth, medical conditions, obstetric complications, parity, presentation and method of delivery, sex of baby, region of residence, oligohydramnios, birth weight, gestation, and plurality.

\section{Main outcome measures}

Development of DDH. Data were reported as odds ratios (ORs) with reference standards mother's age $<20$ years, parity $\geqslant 2$, non-breech presentation, male sex, country living, absence of oligohydramnios, birth weight $3000-3500 \mathrm{~g}$, and gestation 40 weeks.

\section{Main results}

Risks of DDH were associated with many factors (table). In addition, multiple births was associated with decreased DDH (OR 0.06 , CI 0.01 to 0.22 ). When analysed by sex, findings were similar for girls but fewer risk factors were found for boys.

\section{Conclusions}

Many factors were associated with developmental dysplasia of the hip. Greatest risk was associated with breech presentation with breech delivery, breech presentation with caesarean delivery, presence of oligohydramnios, female sex, birth weight $\geqslant 4500 \mathrm{~g}$, and parity 0 for increased risk. Low birth weight, multiple births, and prematurity were protective.

Risk factors for developmental dysplasia of the hip for singleton births

\begin{tabular}{lcl}
\hline Risk factor & Odds ratio & Confidence interval \\
\hline Increased risk & & \\
Maternal age 25-29 years & 1.46 & 1.09 to 1.96 \\
Maternal age 30-34 years & 1.71 & 1.26 to 2.32 \\
Maternal age $\geqslant 35$ years & 1.72 & 1.22 to 2.44 \\
Parity 0 & 2.19 & 1.83 to 2.62 \\
Parity 1 & 1.29 & 1.07 to 1.56 \\
Breech presentation and caesarean & & \\
$\quad$ delivery & 10.0 & 8.58 to 11.7 \\
Emergency caesarean delivery & 13.2 & 10.7 to 16.3 \\
$\quad$ Elective caesarean delivery & 7.56 & 6.27 to 9.12 \\
Breech presentation and delivery & 17.2 & 12.8 to 23.0 \\
Female sex & 3.94 & 3.41 to 4.56 \\
Living in a metropolitan area & 1.45 & 1.23 to 1.69 \\
Living in an outer metropolitan area & 1.86 & 1.41 to 2.46 \\
Presence of oligohydramnios & 3.97 & 1.69 to 9.35 \\
Birth weight 4000-4499 g & 1.55 & 1.26 to 1.91 \\
Birth weight $\geqslant 4500 \mathrm{~g}$ & 2.67 & 1.81 to 3.94 \\
Gestation 41 weeks & 1.26 & 1.05 to 1.51 \\
Gestation $\geqslant 42$ weeks & 1.48 & 1.02 to 2.13 \\
Decreased risk & & \\
Birth weight < 2000 g & 0.30 & 0.12 to 0.77 \\
Birth weight 2000-2499 g & 0.52 & 0.31 to 0.88 \\
Gestation < 37 weeks & 0.42 & 0.25 to 0.69 \\
Gestation 37 weeks & 0.59 & 0.40 to 0.86 \\
Gestation 38 weeks & 0.80 & 0.66 to 0.98 \\
\hline
\end{tabular}

Source of funding: not stated.

For article reprint: Dr A Chan, Epidemiology Branch, South Australian Health Commission, Post Office Box 6, Rundle Mall, Adelaide, South Australia 5000, Australia. Fax +61 882266609.

\section{Commentary}

This study by Chan et al has confirmed the relative importance of previously reported risk factors for $\mathrm{DDH}$ and has identified previously unrecognised risk factors. Although a case control study is not the most rigorous study design, the authors have endeavoured to ensure the accuracy of the results through inclusion of a large number of infants with $\mathrm{DDH}$ (1127 children), confirmed diagnosis by an orthopaedic surgeon (for children with DDH who were born in 1991), inclusion of all eligible controls (150 130 children), and application of sound statistical methods in their risk assessments. All children in the study were, however, born in South Australia and this may limit the generalisability of the results.

The study findings fill a gap in the current research, are clinically relevant, and provide the clinician with greater insight into which children may be at risk of DDH. The study takes previous research further by exploring the association between elective and emergency caesarean section.

DDH is readily treatable. Educational programmes should be offered to child health nurses and physicians. Content should include information on $\mathrm{DDH}$, the importance of early detection, the clinical method of screening, the risk factors, the need for repeated screening for at risk groups until the child is walking, and referral to an orthopaedic surgeon when DDH is suspected. Screening of infants who have identified risk factors does not require special visits at additional costs, but rather should be done before discharge from hospital after birth and at normally scheduled well baby clinic visits.

Brenda Stade, RN, MScN
Clinical Nurse Specialist/
Neonatal Practitioner
Mount Sinai Hospital
Toronto, Ontario, Canada
Emmanuel Quist-Therson, MB
Neonatal Fellow
The Hospital for Sick Children
Toronto, Ontario, Canada
Cinical Nurse Specialist/ Neonatal Practitioner Mount Sinai Hospital therson, MB 